\title{
A Case Study of Persistent, Intense, Clear Air Turbulence in an Upper Level Frontal Zone ${ }^{1}$
}

\author{
RICHARD J. REED \\ Dept. of Atmospheric Sciences, University of Washington, Seatlle \\ AND KenNeth R. HARDY ${ }^{2}$ \\ Air Force Cambridge Research Laboratories, Bedford, Mass. \\ (Manuscript received 21 October 1971, in revised form 10 January 1972)
}

\begin{abstract}
Widespread and persistent clear air turbulence (CAT) occurred over the Eastern Seaboard of the United States between New York and South Carolina on 18 March 1969. The major synoptic features and a qualitative discussion of the factors contributing to the development of the large vertical wind shears associated with the turbulence are presented. The turbulent region in the vicinity of Wallops Island, Va., was probed with a NASA T-33 research aircraft and with sensitive radars. The clear air radar echoes and the most intense turbulence occurred principally within an upper level frontal zone of about $2 \mathrm{~km}$ depth which was produced by the confluence of two currents of widely different origin. The smoothed Richardson number was less than 1.0 throughout the zone and reached its lowest value of $\sim 0.25$ in the region of strongest turbulence. Three distinct types of wave structures were evident in the clear air radar echoes. These were: 1 ) long sinusoidal arches moving at approximately the wind speed which were oriented in the direction of the wind and wind shear and which had wavelengths of $15-30 \mathrm{~km}$ and crest-to-trough amplitudes of nearly 2 $\mathrm{km} ; 2$ ) unstable waves or billows of about $1.6 \mathrm{~km}$ wavelength which were superposed on a portion of the long arches and were also oriented in the shear direction; and 3) braided wave-like patterns having a wavelength of $\sim 5 \mathrm{~km}$ and a crest-to-trough amplitude of more than $1 \mathrm{~km}$ which were oriented in the cross-wind (and cross-shear) direction.
\end{abstract}

\section{Introduction}

Clear air turbulence (CAT) has been the subject of increasing study since it first became a matter of concern in connection with the operation of jet aircraft. Today it is of interest not only because of its effect on flight safety and comfort but also because of its association with atmospheric wave phenomena and its possible importance in the problem of extending the range and accuracy of numerical weather predictions (Panofsky, 1969).

Early investigations of CAT were based largely on reports from commercial and military aircraft. Subsequently, CAT regions have been probed by specially instrumented research aircraft (Endlich and McLean, 1957; Kao and Woods, 1964; Reiter and Burns, 1966; Pinus et al., 1967; Mather, 1969) and more recently by sensitive radars which are capable of detecting the backscatter from turbulence-induced variations in refractive index (Hicks et al., 1967; Glover et al., 1969; Browning and Watkins, 1970; Browning, 1971). In some instances turbulent layers have been observed simultaneously by

\footnotetext{
${ }^{1}$ Contribution No. 251, Department of Atmospheric Sciences, University of Washington.

${ }^{2}$ Much of the research was performed while the co-author was a Visiting Associate Professor in the Department of Atmospheric Sciences, University of Washington.
}

research aircraft and radar (Browning et al., 1970; Glover and Duquette, 1970).

From the observational and theoretical studies carried out during the past several years, and from related studies in the laboratory and the ocean, much progress has been made in describing and understanding CAT (Dutton and Panofsky, 1970). Even so, the subject is still in its infancy, and a continued need exists for observational studies which take advantage of the more sophisticated observing tools now available to describe more fully the structure of CAT and the meteorological conditions which give rise to it.

The object of the present paper is to describe a case of unusual interest which occurred on 18 March 1969 at Wallops Island, Va. On this occasion CAT of considerable intensity and persistence was observed by the NASA T-33 research aircraft in a layer of $2-3 \mathrm{~km}$ in depth, and numerous reports of turbulence were received from commercial aircraft operating in the area. The turbulent region was clearly discernible on the S-band (10.7-cm wavelength) radar which revealed a variety of interesting echo patterns. A cirrus cloud layer above the turbulent region displayed occasional patterns of long, rapidly progressing waves which appeared to be connected with similar waves in the clear air. The description will be based on the radar observations, the 


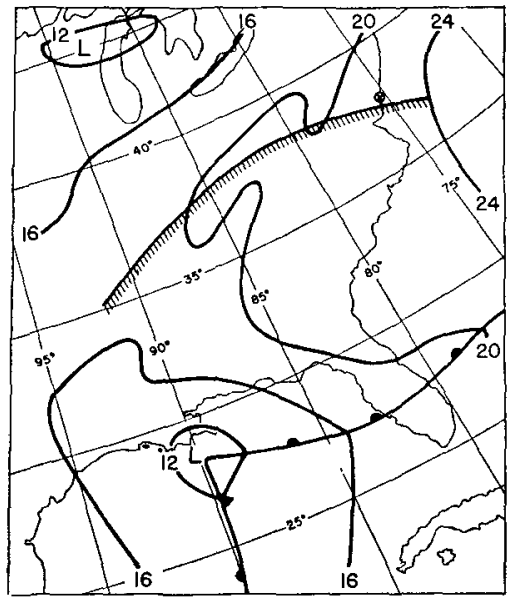

FIg. 1. Surface pressure chart for 0000 GMT 18 March 1969. Note the low center in the Gulf of Mexico which deepened and was associated with the later warm air advection over Wallops Island, Va. $(\otimes)$. Hatching indicates the edge of the cirrus cloud cover.

aircraft measurements of turbulence, and conventional synoptic charts and reports, including a regular rawinsonde taken at Wallops Island shortly before the time of the radar and aircraft observations.

\section{The radar detection of clear air turbulence}

It is now firmly established that sensitive radars with wavelengths $\geqslant 10 \mathrm{~cm}$ often detect echoes which are caused by the backscattering from inhomogeneities of refractive index in the clear atmosphere. The general theory of the scattering of electromagnetic waves by refractive index fluctuations has been treated by Tatarski (1961) and has been confirmed quantitatively by direct experimental observations by Kropli et al. (1968). The radar backscatter is related to the intensity of the fluctuations in refractive index within a very narrow range of eddy sizes which is centered at one-half the radar wavelength. Since the radars at Wallops Island which are used for the turbulence research have wavelengths of 10.7 and $71.5 \mathrm{~cm}$, the eddy sizes responsible for the backscatter will be near 5 and $36 \mathrm{~cm}$, respectively. On the other hand, the turbulence which affects aircraft occurs at scales between about 10 and $1000 \mathrm{~m}$ (Pinus et al., 1967). Assuming that there is inertial transfer of energy through the spectrum from the large to the small eddies according to the Kolmogorov-Obukhov theory, it might be expected that the intensity of turbulent energy at scales affecting aircraft would be reflected in the intensity of turbulent energy at the much smaller scales which are responsible for the radar backscattering. Ottersten (1969) has presented a detailed summary of the radar backscattering from refractive index irregularities and its relationship to atmospheric structure and turbulence. He concluded that the radar reflectivity will be an indicator of the intensity of strong CAT in the stratosphere and, possibly, in the upper troposphere where the contribution of moisture to the vertical refractive index gradient will be small relative to the contribution of temperature. Experiments by Glover et al. (1969) and by Browning et al. (1970) have confirmed the close correlation between the existence of tropospheric clear air radar echoes and turbulence sufficiently intense to be perceived by aircraft. Results of carefully controlled experiments reported by Glover and Duquette (1970) and by Crane (1970) have further verified the consistent detection of CAT by high powered radars.

In addition to the close connection between CAT and the detection of clear air radar echoes, the echoes on many occasions show an organized pattern which may be in the form of waves or billows. On these occasions the echoes provide information on the development of the structures which are responsible for CAT. Hicks and Angell (1968) carried out the first investigation of the wave-like structure observed by radar in the clear atmosphere; this was rapidly followed by other studies of atmospheric waves (Hicks, 1969; Gossard et al., 1970; Atlas et al., 1970; Boucher, 1970; Browning and Watkins, 1970). As described in the following two sections, three types of wave-like echo structures were detected between 0200 and 0400 GMT on 18 March 1969 in the vicinity of Wallops Island. There is evidence that mixing took place over a layer about $2 \mathrm{~km}$ in depth and that waves of quite different wavelength contributed to the widespread moderate turbulence which was reported.

\section{The long waves at cirrus level}

As a result of the warm air and moisture advection from the south, in connection with a deepening wave cyclone in the Gulf of Mexico (Fig. 1), cirrus cloud ap-

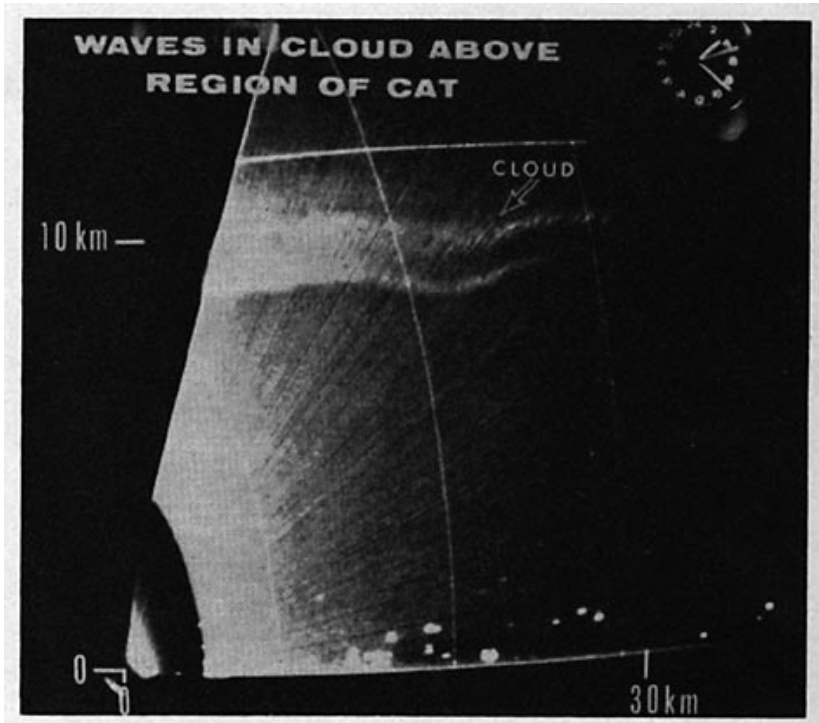

FIG, 2. Photograph of the range-height indicator (RHI) at an azimuth of $270^{\circ}$ for the Wallops Island X-band radar (wavelength of $3.2 \mathrm{~cm}$ ) at $0323 \mathrm{GMT} 18$ March 1969. Range and height marks are indicated in kilometers. The waves are outlined by the scattering from cirrus clouds between 9 and $11 \mathrm{~km}$. 
peared over Wallops Island at about 0200 GMT 18 March. By 0300 long waves were outlined by the radar detection of cirrus cloud. Fig. 2 is a photograph of the range-height indicator (RHI) scope of the $3.2-\mathrm{cm}$ radar and clearly shows the cirrus waves between a height of 9 and $11 \mathrm{~km}$. The radar was scanning in elevation along an azimuth of $270^{\circ}$ or nearly in the upstream direction (Fig. 9b). The 3.2-cm radar at Wallops Island is primarily sensitive to the scattering from cloud and precipitation particles, and detects only the most intense refractive index inhomogeneities which occasionally occur in the lowest few kilometers of the atmosphere. The point or dot echoes in the lowest $1 \mathrm{~km}$ of Fig. 2, however, are due to birds or perhaps insects.

By following the wave pattern from a series of RHI's along the same azimuth, the waves depicted by the cirrus clouds were observed to move eastward with a phase speed of $67 \mathrm{~m} \mathrm{sec}^{-1}$; this is within the range of wind speeds determined from the rawinsonde for the 9-11 $\mathrm{km}$ layer containing the cloud. As shown in the next section (Fig. 4) the winds over Wallops Island at 0300 were estimated to increase from 47 to $71 \mathrm{~m} \mathrm{sec}^{-1}$ in the layer from 9 to $11 \mathrm{~km}$. Using a propagation speed of $67 \mathrm{~m} \mathrm{sec}^{-1}$, it was possible to prepare a composite of the wave pattern from two RHI's taken $180^{\circ}$ from each other but separated in time by $5 \mathrm{~min}$. The results are shown in Fig. 3. At a height of $10 \mathrm{~km}$ the waves have a wavelength of about $17 \mathrm{~km}$ and a crest-to-trough amplitude of about $0.6 \mathrm{~km}$.

Starr and Browning (1972) have reported on the radar observations of several cases of lee waves which ex-
$17 \mathrm{MARCH}, 1969: 2225 \mathrm{EST}$

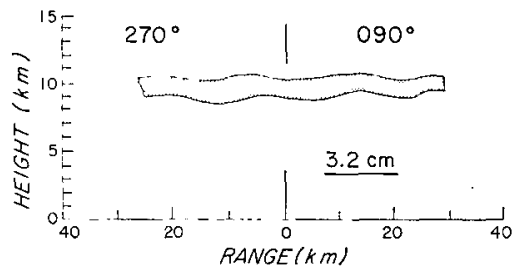

FIG. 3. Composite of two RHI photographs taken at azimuths of $090^{\circ}$ and $270^{\circ}$ with the $3.2-\mathrm{cm}$ radar. The waves are moving with a phase speed of about $67 \mathrm{~m} \mathrm{sec}^{-1}$ which is close to the wind speed at their level. The waves have a wavelength of $17 \mathrm{~km}$ and a crest-to-trough amplitude of $0.6 \mathrm{~km}$.

tended more than $100 \mathrm{~km}$ downstream from the Welsh Mountains. The wavelength of the lee waves varied between 10 and $30 \mathrm{~km}$, and their maximum crest-totrough amplitude was of the order of $800 \mathrm{~m}$. The amplitude generally decreased with height and distance from the mountains. In contrast to the waves shown in Fig. 2 , the waves reported by Starr and Browning either moved very slowly or were stationary over periods as long as $5 \mathrm{hr}$. Thus, it is evident that the waves seen in Fig. 2 are not lee waves, but appear to be imbedded within the mean flow.

\section{The wave structure of clear air radar echoes}

The major radar echoes in the clear atmosphere during the period from 0200-0400 GMT occurred at a height between about 7 and $9 \mathrm{~km}$. From the nearly northsouth vertical cross section depicted in Fig. 4, it is evi-

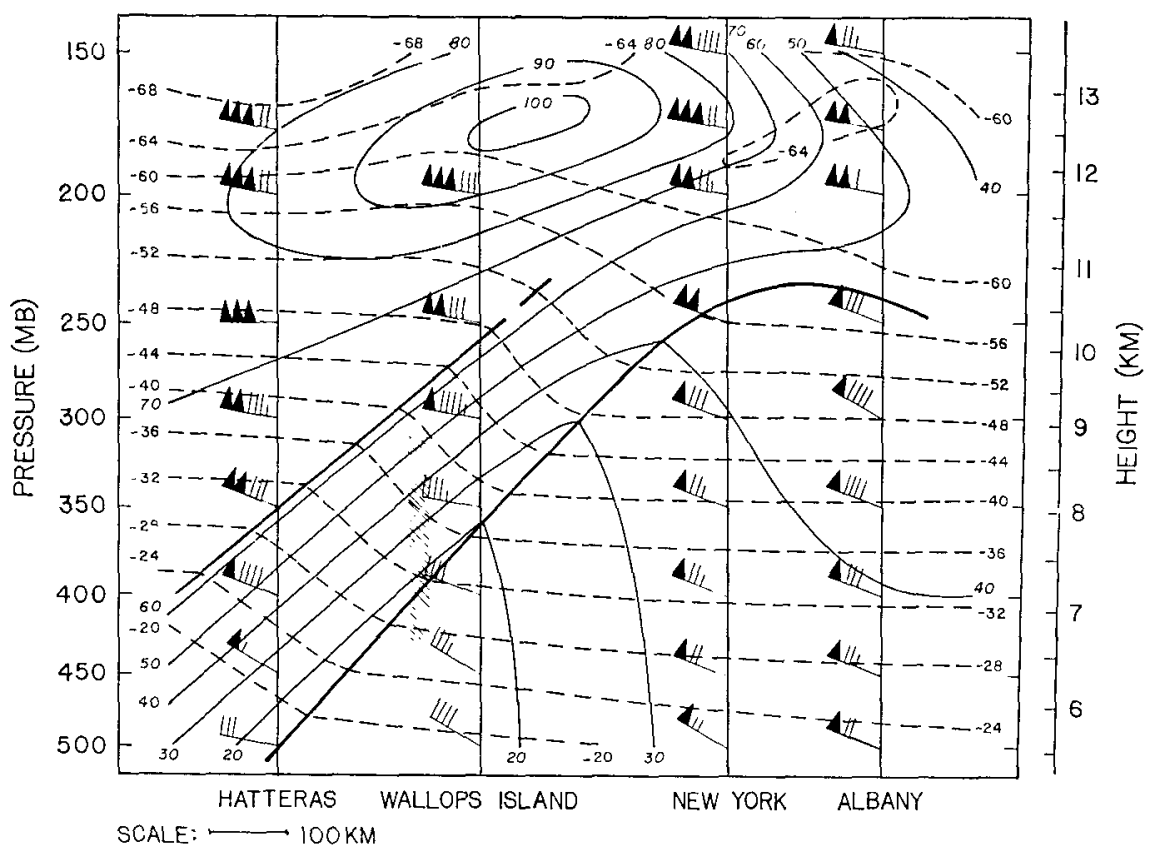

FIG. 4. A vertical cross section between Albany, N. Y., and Hatteras, N. C., for 0000 GMT 18 March 1969. The main baroclinic zone is marked by heavy solid lines. The isotachs of normal wind component $(\mathrm{m} \mathrm{sec}-1)$ are solid lines and the isotherms $\left({ }^{\circ} \mathrm{C}\right.$ ) are dashed (a full barb is $5 \mathrm{~m} \mathrm{sec}^{-1}$; a flag, $25 \mathrm{~m} \mathrm{sec}-1$ ). The radar layers (indicated by shading) and the strongest CAT occurred within the cloud-free frontal zone. 
dent that the echoes were received predominantly from an upper level frontal zone which was situated in the area at that time. In locating the echoes on the cross section, allowance was made for the northward displacement of the front during the interval between the radiosonde and radar observations in order that the crosssection data and radar data could be compared. The radar patterns gave abundant evidence of disturbed flow and peculiar structures. However, as shown in Figs. 5 and 6, there were two occasions when the echo structures probably were outlining large secondary billows which

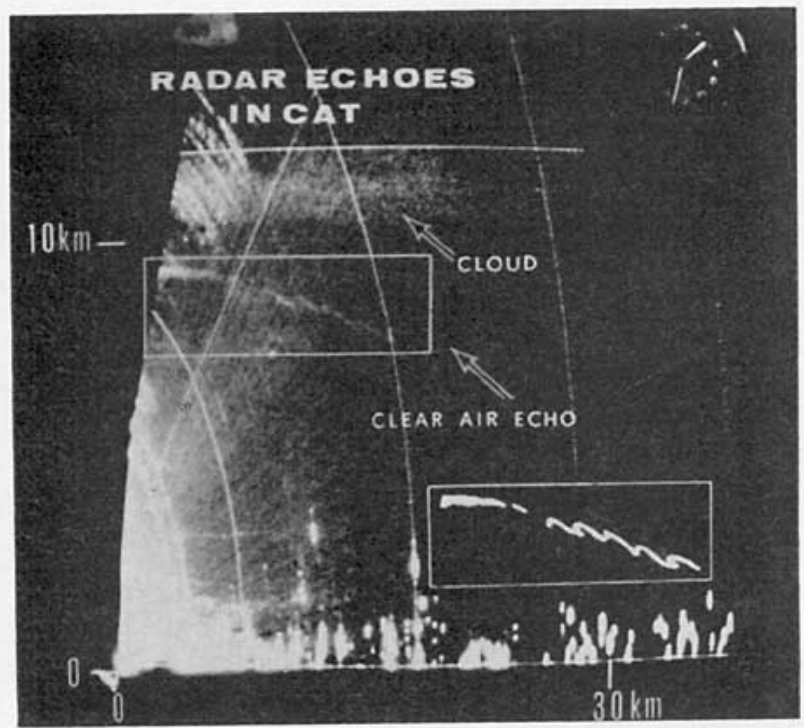

FIG. 5. Photograph of the RHI at a wavelength of $10.7 \mathrm{~cm}$, for 0332 GMT 18 March 1969 (aximuth $270^{\circ}$ ). The wind direction and shear are toward the left. Note the portion of the long arch and the superposition of the billow structure. The verticle scale is expanded by a factor of $\sim 3$, accounting for the seemingly large slope of the long wave. The inset is a tracing from the original record.

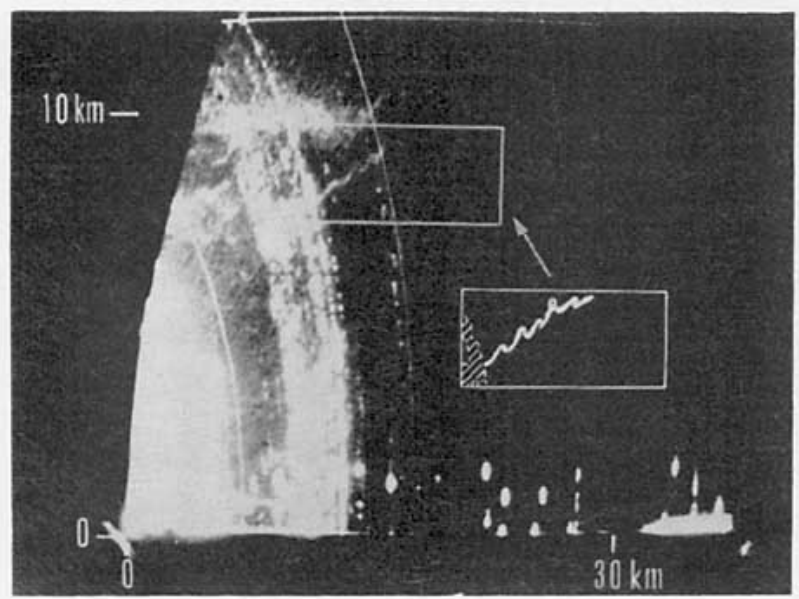

FIG. 6. Same as Fig. 5 except for a time of 0314 GMT and an azimuth of $090^{\circ}$. Wind direction and shear are toward the right. Looking downwind, the billow structure again forms along the upward sloping portion of the long wave. For a wind increasing with height, this is the portion of the wave where the vorticity is concentrated. formed on the tilted portion of the long waves (Ludlam, 1967; Scorer, 1969a). In both cases the billows appear on the upward slope looking downwind of a longer wave (radar azimuth $270^{\circ}$ in Fig. 5 and $090^{\circ}$ in Fig. 6). The billows have a wavelength of $\sim 1.6 \mathrm{~km}$ and a crest-totrough amplitude of $\sim 200 \mathrm{~m}$. Since the wind shear is toward the east (Fig. 4), the billows have formed on the portion of the wave where the vorticity is concentrated (Scorer, 1969a).

Only about one-half a wavelength of the longer waves are depicted by the clear air radar echoes of Figs. 5 and 6 . With use of these admittedly incomplete observations, the wavelength and crest-to-trough amplitude of the long waves in the clear air are found to be at least 30 and $1.8 \mathrm{~km}$, respectively, in Fig. 5 , and 15 and 1.5 $\mathrm{km}$, respectively, in Fig. 6 . These are minimum estimates because the locations of the troughs or peaks of the waves are not clearly discernible. The $15-\mathrm{km}$ wavelength compares closely with that observed at the cirrus level. In a theoretical study of the stability of a shear layer, Miles and Howard (1964) found a ratio of 7.5 for the ratio of wavelength to layer depth for the wavelength of maximum instability. In the present case with a $2-\mathrm{km}$ depth of the frontal layer, the theoretically predicted wave length is $15 \mathrm{~km}$, in close agreement with the observed length of most of the longer waves seen by radar.

From two RHI's taken along $270^{\circ}$ and separated by $3 \mathrm{~min}$, it was possible to estimate the speed of the long waves within the clear air at a height of $8.3 \mathrm{~km}$. Using identifiable features of the wave on both RHI's, it was found that the wave propagated at $44 \mathrm{~m} \mathrm{sec}^{-1}$. The wind at $8.3 \mathrm{~km}$ within the region of the frontal zone con-

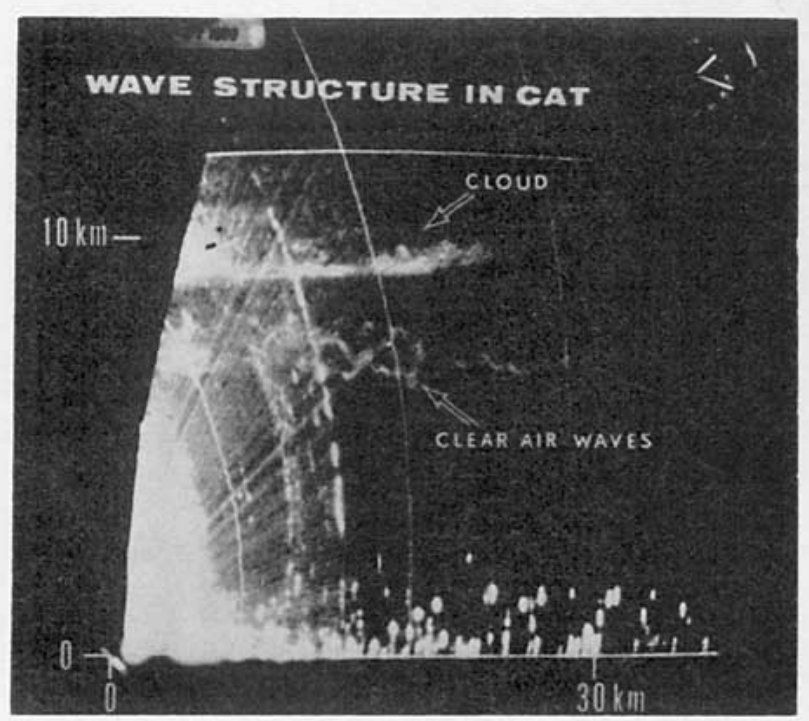

FIG. 7. Same as Fig. 5 except for a time of 0318 GMT and an azimuth of $0^{\circ}$. The perturbed clear air pattern displays evidence of a periodic structure. Since the $0^{\circ}$ azimuth is normal to the wind and shear direction, the stream surface is corrugated or perturbed in a direction parallel to the wave fronts of the long waves. 


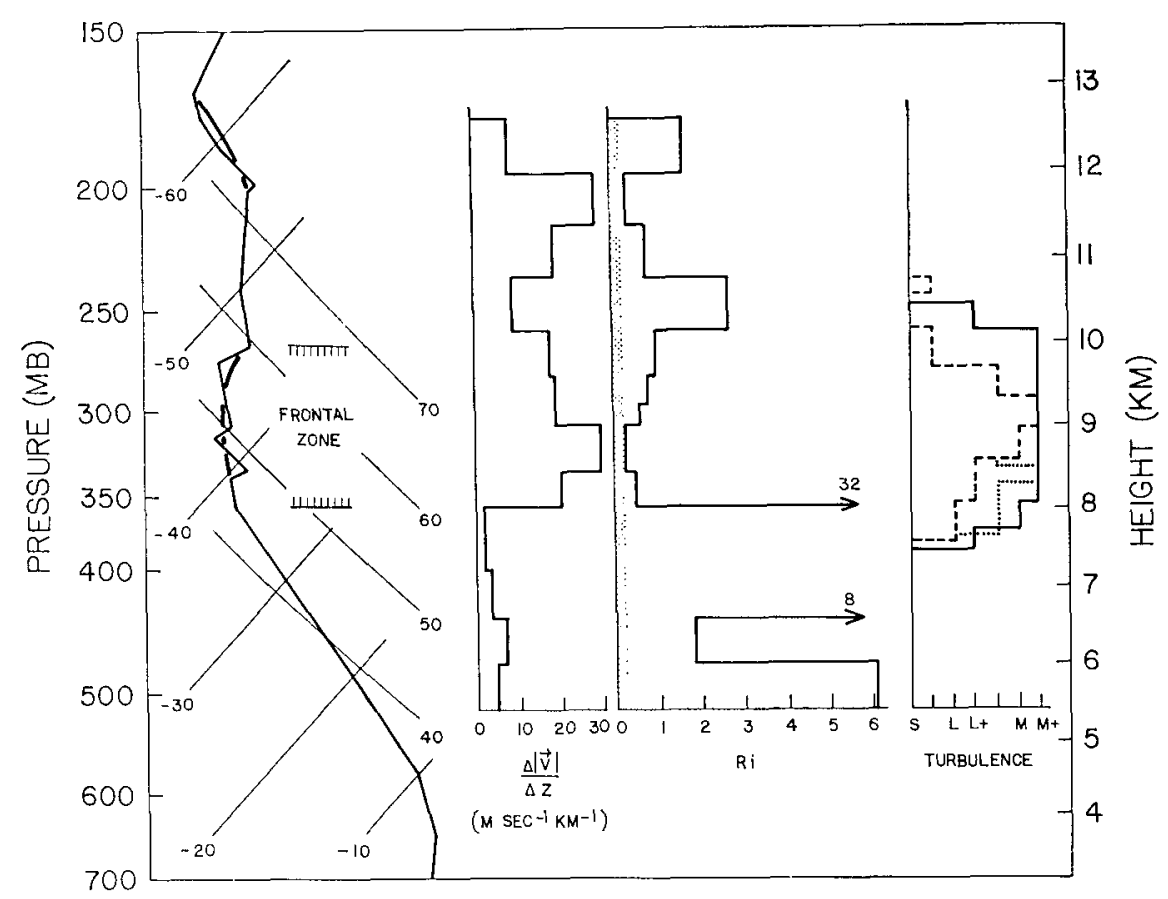

FIG. 8. Temperature, vector wind shear, and Richardson number (Ri) based on the 0000 GMT 18 March 1969 sounding at Wallops Island. Also plotted are the pilot's estimates of turbulence intensity for the NASA T-33 research aircraft during three traverses through the layer which were flown between 0230 and 0300 GMT. The letters S, L and M denote smooth, light and moderate turbulence, respectively, and the plus sign denotes an intermediate category. Note the coincidence of the turbulence with the low Ri and the small-scale temperature perturbations. Heavy segments of temperature sounding denote smoothed values used in computing $\mathrm{Ri}$.

taining the clear air echoes was about $45 \mathrm{~m} \mathrm{sec}^{-1}$ (Fig. 4). While this is excellent agreement, the experimental errors are estimated to be about $\pm 10 \%$. However, within the measurement error, the clear air waves, like the waves at the higher cirrus level, were moving with the speed of the wind appropriate for their altitudes.

Much of the wave structure detected in the clear atmosphere by radar has been interpreted as being due to billows which are characterized by wave fronts normal to the wind shear (Browning, 1971). However, for almost 10 min on $18 \mathrm{March}$, complex periodic structures were detected at right angles to the shear vector and thus could not be a form of billows. An example of such an echo pattern at an azimuth of $0^{\circ}$ is shown in Fig. 7 . The echoes are somewhat chaotic, but there is evidence of some type of instability which is organized in a wavelike pattern. Although difficult to define, the most pronounced portion of the wave pattern has a wavelength of $\sim 5 \mathrm{~km}$ and a crest-to-trough amplitude $>1$ $\mathrm{km}$. Scorer (1969b) discusses centrifugal instability which is manifested by the production of vorticity with its axis along the streamlines, as a possible CAT mechanism. Thus, disturbances arising from centrifugal instability could effectively form corrugations of the stream surfaces in a direction which is nearly parallel to the wave fronts of the long waves. For velocity increasing with height (Fig. 4), centrifugal instability would form in wave troughs of the long waves. With the present radar data, however, it is not possible to determine whether the conditions are conducive for the existence of centrifugal instability. It is mentioned only to indicate that it is a mechanism which can lead to periodic structures which are oriented at large angles to the primary wave fronts. The existence of these apparent crossed wave patterns is also evident in some clouds which may exhibit periodic structures which are nearly perpendicular to the wave fronts of the primary waves.

\section{Aircraft turbulence}

As shown in Fig. 8, the NASA T-33 aircraft encountered extensive turbulence between 7.5 and $10.5 \mathrm{~km}$ as the pilot passed through this layer on three traverses. The pilot did not fly above a height of $10.8 \mathrm{~km}$, and no turbulence was encountered below a height of $7 \mathrm{~km}$. The aircraft flew east of the radar site, and the pilot estimated turbulence intensities of moderate or greater over a period of more than $30 \mathrm{~min}$. In fact, the pilot had flown many turbulence missions, and he reported that the turbulence on this occasion was the most severe he had ever experienced on the East Coast. From the accompanying temperature sounding it is evident that the turbulence was strongest, on the average, in the 
TABLE 1. Turbulence encountered by commercial aircraft between Wallops Island and Gordonsville, Va., between 1800 GMT 17 March and 0600 GMT 18 March 1969.

\begin{tabular}{|c|c|c|c|c|c|}
\hline Area & None & $\begin{array}{l}\text { Turbs } \\
\text { Very light } \\
\text { to light }\end{array}$ & $\begin{array}{l}\text { Lilence inte } \\
\text { moderate }\end{array}$ & $\begin{array}{l}\text { nsity } \\
\text { Moderate } \\
\text { to severe }\end{array}$ & $\begin{array}{l}\text { Percent } \\
\text { of reports } \\
\text { with } \\
\text { turbulence }\end{array}$ \\
\hline \multirow{2}{*}{$\begin{array}{l}\text { Above frontal } \\
\text { zone } \\
\text { In frontal zone } \\
\text { Below frontal } \\
\text { zone }\end{array}$} & $\begin{array}{l}8 \\
2\end{array}$ & $\begin{array}{r}11 \\
8\end{array}$ & $\begin{array}{l}0 \\
1\end{array}$ & $\begin{array}{l}0 \\
2\end{array}$ & $\begin{array}{l}58 \\
85\end{array}$ \\
\hline & 2 & 0 & 0 & 0 & 0 \\
\hline
\end{tabular}

middle of the frontal zone and that it encompassed the entire zone and extended somewhat into the adjacent regions. Although the pilot did not probe the region above $11 \mathrm{~km}$, it is clear from the radar data and reports from commercial aircraft that turbulence also existed well above the frontal zone.

Also shown in Fig. 8 are vertical profiles of the temperature, total wind shear, and the Richardson number based on the 0000 GMT sounding for Wallops Island. The shear was computed from the original rawinsonde data sheet which gives average winds in $\sim 600 \mathrm{~m}$ layers (2 min averages). For consistency, static stabilities for the same layers were used in computing the Richardson number. The smoothed temperature curve used to compute these stabilities is depicted by the thick line segments in Fig. 8. The actual temperature sounding, taken from the original record (thin line), shows smaller scale temperature fluctuations which were probably associated with the turbulence and wave activity. It seems likely that some of the echo filaments seen in Figs. 5-7 are associated with these or similar fine-scale features.

The measurements reveal that the Richardson number was less than unity throughout the depth of the frontal zone and that it decreased to 0.25 in the interior of the zone where the temperature trace indicates an adiabatic layer bounded on either side by thin inversion layers. It is evident that the turbulence observed by the research aircraft reached its peak intensity very close to the level of minimum Richardson number. Browning (1971) found that large-amplitude billows, as observed in the clear air by radar, were also associated with Richardson numbers which were in the range of $0.15-0.3$ when evaluated over layers $200 \mathrm{~m}$ in depth.

The frontal zone is surmounted by a layer of increased Richardson number $(\sim 3)$ above which it decreases again to a value near 0.25 . This low value is connected with the strong shear below the jet core where the horizontal temperature gradient is small but the anticyclonic curvature is near the critical value for gradient flow. This point will be discussed further in the next section. In general, the profile of the Richardson number parallels that of the wind shear, variations of static stability playing only a secondary role in its behavior.

Turbulence was quite widespread and was encountered by commercial aircraft in the vicinity. Summarized in Table 1 are pilot reports from all Eastern Airlines flights operating in the area between Wallops Island and Gordonsville, Va., during the period 1800 GMT March 17 to 0600 GMT March 18. From the table it is seen that turbulence was much more likely to occur throughout the frontal zone and that all cases of moderate-tosevere intensity occurred within it. In the warm air mass above the zone light turbulence was encountered on about half the flights. Conceivably, some of this turbulence could have occurred in the cirrus cloud layer, although cases were excluded in which specific mention was made of cloud.

\section{Synoptic setting}

In view of the persistent and widespread nature of the turbulence, it is of interest to examine the synoptic conditions associated with the development of the upper level frontal zone and with the production and maintenance of the low Richardson numbers which characterized the zone. Roach (1970) has derived an index for the development of low Richardson numbers by synoptic-scale motions which would appear to have application in this connection. According to this index, stretching deformation, with the axis of dilatation normal to the vertical shear vector (approximately the direction of the temperature gradient) and vertical shear of the ageostrophic wind component in the direction from warm to cold air, contribute to the development of low Richardson numbers. In the present case, however, preliminary measurements revealed that these two effects were of comparable magnitude and of opposite sign, the ageostrophic shear being of the proper sense to produce low values but the stretching deformation being of the opposite sense. Since it appeared doubtful that the data were sufficiently accurate to evaluate a residual quantity (the difference between two large terms), we have not attempted a quantitative study of synoptic factors underlying the turbulent outbreak. Instead, we will confine our further remarks to a description of the major synoptic features and to a mainly qualitative discussion of the factors associated with the development of the large vertical wind shears which were primarily responsible for the observed low Richardson numbers.

The upper level flow patterns at 1200 GMT 17 March and at the time of the radar observations $12 \mathrm{hr}$ later are shown in Figs. 9a and b. At the later hour Wallops Island lies very close to the line of confluence between two currents of widely different origin - a nearly straight current which crosses the northern United States and dips southward across the Eastern Seaboard, and a much faster, anticyclonically curved current from lower latitudes which a short time earlier has passed to the south of a sharp trough and associated closed low over the central United States. The strong ageostrophic flow upstream of the ridge can be seen visually from the angle between the height contours and the plotted winds. 


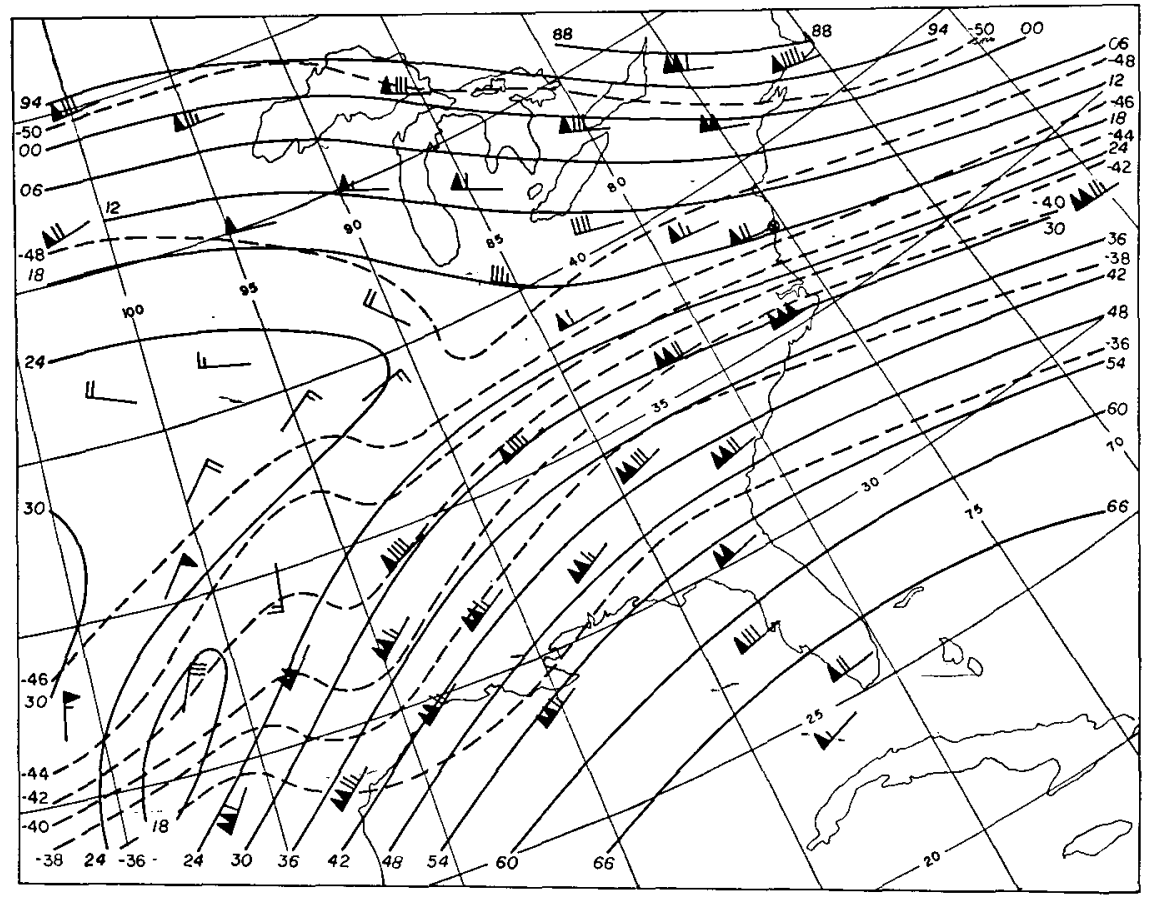

a.

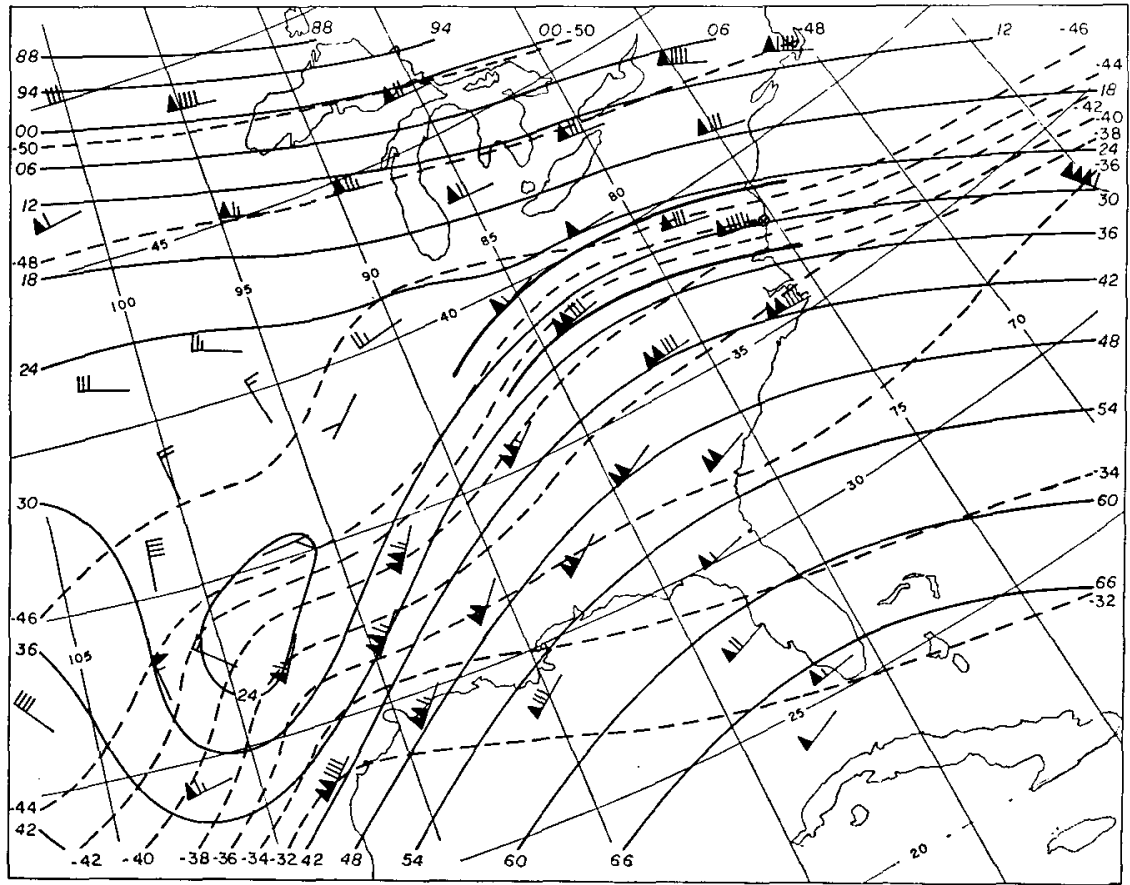

b.

FIG. 9. The 300-mb charts for 1200 GMT 17 March, a., and 0000 GMT 18 March 1969, b. The frontal zone at 0000 GMT in the vicinity of Wallops Island $(\otimes)$ is indicated by thick lines. Contours are solid lines and are labeled in tens of meters. Isotherms $\left({ }^{\circ} \mathrm{C}\right)$ are dashed. Note the strong ageostrophic flow upstream of the ridge at 0000 GMT as indicated by the angle between the contours and the plotted winds (a full barb is $5 \mathrm{~m} \mathrm{sec}^{-1}$; a flag, $25 \mathrm{~m} \mathrm{sec}-1$ ). 
The frontal zone, which was viewed earlier in cross section (Fig. 4), is well marked on both 300 -mb charts, advancing northward towards Wallops Island during the 12-hr interval. If horizontal trajectories of air parcels located at the warm and cold edges of the frontal zone in the vicinity of Wallops Island at the later hour are traced upstream (backward in time) to their points of origin, it is evident from the upstream temperatures, in the absence of vertical motions, that the temperature difference across the zone would have been larger than observed. Slight warming, indicative of weak subsidence, occurred along trajectories on the cold side of the zone; marked cooling and ascent occurred along trajectories on the warm side. The cirrus cloudiness in the warm air just above the frontal zone affords visible evidence of the uplift. The frontal zone was clearly maintained at nearly constant strength by the opposing eff ects of horizontal confluence acting to strengthen the temperature gradient and a direct circulation in the meridional plane normal to the flow which operated to reduce it. An additional factor tending to counteract the frontogenetical process was the turbulent mixing in the zone.

A further interesting point to note is that the thermal wind shear corresponding to the actual temperature gradient in the vicinity of Wallops Island at $300 \mathrm{mb}$ and in the cross section (Fig. 4) is only about one-half the observed wind shear and, therefore, far short of the shear required to yield the critical Richardson number of 0.25 at which wave instability begins. Since the flow is anticyclonic in and to the south of (or above) the frontal zone, it seems reasonable to ascribe the difference between the thermal and observed wind shears to a state of gradient rather than geostrophic wind balance. From differentiation of the gradient wind equation with respect to height $z$, the gradient wind shear may be written as

$$
\left(\frac{\partial v}{\partial z}\right)_{\mathrm{grad}}=\left[f\left(\frac{\partial v}{\partial z}\right)_{\mathrm{geo}}-v_{\mathrm{grad}}^{2} \frac{\partial K}{\partial z}\right] /\left(2 K v_{\mathrm{grad}}+f\right),
$$

where $v$ is the wind speed, $f$ the Coriolis parameter, $K$ the curvature of the flow, and the subscripts refer to gradient and geostrophic winds. The results of evaluating this shear in three layers, two within the frontal zone and one above, and comparison with the actual and thermal wind shears are given in Table 2. The comparison indicates that anticyclonic curvature could ac- count for the difference between the actual and geostrophic wind shears. In the lower portion of the frontal zone a substantial part of the extra contribution to the shear is derived from the increase of anticyclonic curvature with height. In the upper part of the zone, and in the layer above, the dominant effect is the reduction of the denominator due to the combined effect of large wind speeds and negative (anticyclonic) curvature of the flow. In the upper layer only a small temperature gradient is required to give a large shear. It is evident from this result that regions of large shear not directly connected with frontal structure may exist in the vicinity of anticyclonically curved jet streams.

\section{Summary and conclusions}

Persistent, widespread turbulence of light-to-moderate intensity occurred in an upper tropospheric frontal zone of about $2 \mathrm{~km}$ in depth. Although the temperature gradient was unusually strong for such a zone, it was not sufficiently large to reduce the Richardson number to its critical value $(0.25)$ for wave instability through thermal wind shear alone. Evaluation of the gradient wind shear revealed that the effects of anticyclonic curvature of the flow contributed equally to the observed shear. The frontal zone was produced by a confluent flow pattern and would have been much more intense except for the counter effect of a direct circulation in the meridional plane normal to the flow. This meridional circulation was characterized by weak sinking motion on the cold side of the zone, rising motion on the warm side, and an upward increasing ageostrophic wind component from the warm to cold air side.

In addition to the turbulence within the frontal zone, commercial aircraft reported the frequent occurrence of light turbulence in the region above this zone. Large vertical wind shears and low Richardson numbers were also observed in this region despite the small thermal gradients, a condition which it was shown can exist in strong anticyclonic flow when the curvature is close to the critical value for gradient wind balance.

Though generally chaotic in appearance, the clear air echo pattern in the frontal zone at times showed organized structures. These were of three types: 1) long sinusoidal arches in the direction of the wind and the shear, describing waves of $15-30 \mathrm{~km}$ wavelength and crest-to-trough amplitudes of nearly $2 \mathrm{~km} ; 2$ ) unstable

TABLE 2. Comparison of geostrophic, gradient and actual wind shear.

\begin{tabular}{|c|c|c|c|}
\hline $\begin{array}{l}\text { Layer } \\
(\mathrm{mb})\end{array}$ & $\begin{array}{c}\left(\frac{\partial v}{\partial z}\right)_{\mathrm{geo}} \\
\left(\mathrm{m} \mathrm{sec} \mathrm{km}^{-1}\right)\end{array}$ & $\begin{array}{l}{\left[f\left(\frac{\partial v}{\partial z}\right)_{\mathrm{geo}}-v_{\mathrm{grad}}^{2} \frac{\partial K}{\partial z}\right] /\left[2 K v_{\mathrm{grad}}+f\right]=\left(\frac{\partial v}{\partial z}\right)_{\mathrm{grad}}} \\
\left(10^{-7} \mathrm{cgs} \text { units }\right)\left(10^{-5} \mathrm{cgs} \text { units }\right)\left(\mathrm{m} \mathrm{sec}^{-1} \mathrm{~km}^{-1}\right)\end{array}$ & $\underset{\left(m \sec ^{-1} \mathrm{~km}^{-1}\right)}{\left(\frac{\partial v}{\partial z}\right)_{\text {sctual }}}$ \\
\hline $\begin{array}{l}360-300 \\
300-260 \\
250-200\end{array}$ & $\begin{array}{l}7.9 \\
9.5 \\
3.6\end{array}$ & $\begin{array}{l}(7.2+4.4) /(-1.5+9.0)=15.5 \\
(8.6+0) /(-4.9+9.0)=21.0 \\
(3.3+0) /(-6.8+9.0)=15.0\end{array}$ & $\begin{array}{l}18.5 \\
20.0 \\
17.3\end{array}$ \\
\hline
\end{tabular}


billows of about $1.6 \mathrm{~km}$ wavelength, also oriented in the direction of the shear, which appeared on the upward slope of the long arches viewed downwind; and 3) braided, wave-like patterns in the cross wind (and shear) direction with wavelengths of about $5 \mathrm{~km}$ and crest-totrough amplitudes of about $1 \mathrm{~km}$.

The long wave pattern moved with approximately the mean wind in the layer. The cirrus cloud layer above the frontal zone also exhibited long wavelike perturbations which propagated with the wind. The ratio of the depth of the frontal zone $(2 \mathrm{~km})$ to the length of most of the long waves $(15-20 \mathrm{~km})$ is in reasonable accord with the theoretically predicted value of $1: 7.5$ (Miles and Howard, 1964). It is speculated that the braided patterns in the cross-shear direction are manifestations of the centrifugal instability discussed by Scorer (1969b).

The present study points out anew the diversity and complexity of the echo patterns and atmospheric structures associated with CAT. Many more studies involving sensitive radar, specially instrumented aircraft, and conventional observing systems will be needed before CAT can be fully described and understood.

Acknowledgments. The research conducted at Wallops Island has been supported in part by the National Aeronautics and Space Administration. The initiative to acquire radar data on this occasion was due entirely to Jack Howard, and we wish to thank him and other personnel of the Applied Physics Laboratory, Johns Hopkins University, for their careful conduct of the experiment. We thank Robert L. Krieger and his staff at NASA Wallops Station and Joe Stickle of the NASA Langley Research Center for their cooperation and support of the experiment. Appreciation is also expressed to Paul W. Kadlec of Continental Airlines (formerly with Eastern Airlines) for his collection of aircraft CAT reports, to Margaret Lemone, Hans Ottersten and Dr. Alistair B. Fraser for their helpful discussions during the preparation of the paper, to Ralph J. Donaldson, Jr., and Isadore Katz for their critical reading of the paper, and finally to the following graduate students in the Department of Atmospheric Sciences, University of Washington for their assistance in the synoptic analyses: Roger Andersen, Terry Fox, Bob Pratt and John Shipe. The research activities of one of the authors (R.J.R.) was supported by the National Science Foundation under Grant GA629X.

\section{REFERENCES}

Atlas, D., J. I. Metcalf, J. H. Richter and E. E. Gossard, 1970: The birth of "CAT" and microscale turbulence. J. Atmos. Sci., 27, 903-913.

Boucher, R. J., 1970: CAT at a subsidence inversion : A case study. J. Appl. Meteor., 9, 534-537.

Browning, K. A., 1971: Structure of the atmosphere in the vicinity of large-amplitude Kelvin-Helmholtz billows. Quart. J. Roy Meteor. Soc., 97, 283-299.
, and C. D. Watkins, 1970: Observations of clear air turbulence by high power radar. Nature, 227, 260-263.

- - - - J. R. Starr and A. McPherson, 1970: Simultaneous measurements of clear air turbulence at the tropopause by high-power radar and instrumented aircraft. Nature, 228, 1065-1067.

Crane, R. K., 1970: Measurement of clear air turbulence in the lower stratosphere using the Millstone Hill L-Band radar. Preprints of Papers, Fourteenth Radar Meteor. Conf., Tucson, Ariz., Amer. Meteor. Soc., 101-106.

Dutton, J. A., and H. A. Panofsky, 1970: Clear air turbulence: A mystery may be unfolding. Science, 167, 937-944.

Endlich, R. M., and G. S. McLean, 1957: The structure of the jet stream core. J. Meteor., 14, 543-552.

Glover, K. M., R. J. Boucher, H. Ottersten and K. R. Hardy, 1969: Simultaneous radar, aircraft, and meteorological investigations of clear air turbulence. J. Appl. Meteor., 8, 634-640.

- - and E. F. Duquette, 1970: A study of clear air turbulence using sensitive radars. Preprints of Papers, Fourteenth Radar Meteor. Conf., Tucson, Ariz., Amer. Meteor. Soc., 89-94.

Gossard, E. E., J. H. Richter and D. Atlas, 1970: Internal waves in the atmosphere from high-resolution radar measurements. J. Geophys. Res., 75, 3523-3536.

Hicks, J. J., 1969: Radar observations of a gravitational wave in clear air near the tropopause associated with CAT. $J . A p p l$. Meteor., 8, 627-633.

- $\longrightarrow$, and J. K. Angell, 1968: Radar observations of breaking gravitational waves in the visually clear atmosphere. $J . A p p l$. Meteor., 7, 114-121.

- , I. Katz, R. R. Landry and K. R. Hardy, 1967: Simultaneous radar and aircraft observations of clear-air turbulence. Science, 157, 808-809.

Kao, S.-K., and H. D. Woods, 1964: Energy spectra of mesoscale turbulence along and across the jet stream. J. Atmos. Sci., 21, 513-519.

Kropfli, R. A., I. Katz, T. G. Konrad and E. B. Dobson, 1968 : Simultaneous radar reflectivity measurements and refractive index spectra in the clear atmosphere. Radio Sci., 3, 991-994.

Ludlam, F. H., 1967: Characteristics of billow clouds and their relation to clear air turbulence. Quart.J. Roy. Meteor. Soc., 93, 419-435.

Mather, G. K., 1969: Clear air turbulence research activities at the National Aeronautical Establishment. Symposium on Clear Air Turbulence and its Detection, New York, Plenum Press, 271-287.

Miles, J. W., and L. N. Howard, 1964: Note on a heterogeneous shear flow. J. Fluid. Mech., 20, 331-336.

Ottersten, H., 1969: Atmospheric structure and radar backscattering in clear air. Radio Sci., 4, 1179-1193.

Panofsky, H. A., 1969: Internal atmospheric turbulence. Bull. Amer. Meteor. Soc., 50, 539-543.

Pinus, N. Z., E. R. Reiter, G. N. Shur and N. K. Vinnichenko, 1967: Power spectra of turbulence in the free atmosphere. Tellus, 19, 206-213.

Reiter, E. R., and A. Burns, 1966: The structure of clear-air turbulence derived from "TOPCAT" aircraft measurements. $J$. Atmos. Sci., 23, 206-212.

Roach, W. T., 1970: On the influence of synoptic development on the production of high level turbulence. Quart.J. Roy. Meteor. Soc., 96, 143-429.

Scorer, R. S., 1969a: Billow mechanics. Radio Sci., 4, 1299-1307.

- $1969 \mathrm{~b}$ : Mechanisms of clear air turbulence. Symposium on Clear Air Turbulence and its Detection, New York, Plenum Press, 34-50.

Starr, J. R., and K. A. Browning, 1972: Observations of lee waves by high-power radar. Quart. J. Roy. Meteor. Soc., 98 (in press).

Tatarski, V. I., 1961 : Wave Propagation in a Turbulent Medium. New York, McGraw-Hill, 285 pp. 